\title{
¿QUÉ TANTO ENTENDEMOS EL CONCEPTO DE PRÁCTICA BASADA EN LA EVIDENCIA EN TERAPIA OCUPACIONAL?
}

\section{José Miguel Aravena Castro ${ }^{1}$}

Los procesos de intervención y de razonamiento clínico requieren de una renovación constante y de una mirada crítica de los procedimientos que se realizan. La práctica basada en la evidencia (EBP) surge como un método que busca integrar el conocimiento emergente de la evidencia científica, con el conocimiento que tiene el propio profesional, y las necesidades del sujeto en un contexto determinado, con el fin de entregar una atención más atingente y de calidad a la persona, buscando obtener los mejores resultados para el usuario, a través del método más adecuado para su situación específica (Evidence-Based Medicine Working Group, 1992). En la práctica de la Terapia Ocupacional, han surgido diversos debates en relación a la utilización de la EBP (conocida como Terapia Ocupacional Basada en la evidencia -TOBE-), puesto que no existe una definición establecida o un arraigo propio de la disciplina de este método en su práctica habitual (Cohn, Coster, \& Kramer, 2014), poniendo en tensión o en duda de forma frecuente la real validez, efectividad y replicabilidad de la intervención de Terapia Ocupacional en los diversos escenarios de acción.

El tema se resume de buena forma a través de una revisión sistemática publicada en el año 2014 en la British Occupational Therapy Journal (Upton, Stephens, Williams, \& Scurlock-Evans, 2014). Este estudio buscó conocer las percepciones, actitudes e implementación de la práctica basada en la evidencia (EBP) por parte de los propios Terapeutas Ocupacionales. Para esto se realizó una búsqueda de artículos con metodologías cua-

Terapeuta Ocupacional, Licenciado en Ciencias de la Ocupación Humana, Candidato a Magister en Envejecimiento y Calidad de vida, Universidad de Chile. Mail: jm.aravenac@gmail.com. litativas y cuantitativas publicados entre el año 2000 y 2012, que contuvieran entre sus palabras claves: conocimiento, entendimiento, práctica clínica, actitudes, implementación, evidencia, entre otras. Todo esto en el contexto de la Terapia Ocupacional.

De los 12.990 artículos elegibles, 32 cumplieron los criterios de inclusión, de entre los cuales, como principales hallazgos se encontró que los Terapeutas Ocupacionales en su mayoría presentan una buena actitud acerca del concepto de EBP y de su eventual importancia. Sin embargo, existe una proporción importante de sujetos que tienen una percepción negativa. Dicha percepción estaba relacionada con los años de especialización (mientras más años más positivo y a la inversa), área de trabajo (académica resulto ser más positiva), lugar de trabajo (zona rural presentó mayor percepción negativa), y año de egreso (en jóvenes es más positiva). En cuanto al uso de la EBP, se observó que en la mayoría de los estudios los profesionales preferían solo usar su experiencia personal y juicio clínico y verificar resultados caso a caso, manifestándose como principales razones para no utilizar la EBP: la carga laboral, el tiempo que requiere el proceso, recursos insuficientes, falta de entrenamiento o habilidades, barreras institucionales, relevancia, aplicabilidad y calidad de los estudios, reportándose solo un $12 \%$ de utilización de la EBP en uno de los estudios. En contraste con esto, los TO manifestaban la importancia de implementar la EBP y de adquirir herramientas para mejorar sus habilidades, expresando la necesidad de generar espacios que permitan adquirir las competencias para su implementación.

En relación a la revisión, si bien este estudio posee algunas limitaciones metodológicas a considerar para futuras revisiones sistemáticas, como una escasa definición de los criterios de inclusión de los estudios esco- 
gidos, lo cual dificulta la comparación y generalización de los estudios y sus hallazgos, los resultados encontrados arrojan ciertas tendencias que permiten generar ideas y una visión crítica en relación a las percepciones y actitudes de los Terapeutas Ocupacionales hacia la EBP.

Un factor influyente, según los autores, es la definición y entendimiento que se tiene de la EBP desde la $\mathrm{TO}$, debido a que según los reportes un gran número de los sujetos no tiene muy claro hacia dónde apunta el concepto de la EBP, por lo que el conocimiento profesional y experiencial se convierte en la única herramienta de apoyo para la toma de decisiones clínicas y definiciones de tratamiento ante situaciones específicas. En relación a esto, surge la pregunta ¿Cómo profesionales de la salud, los Terapeutas Ocupacionales entendemos realmente lo que es la EBP?

La EBP en Terapia Ocupacional es el proceso de búsqueda sistemática, evaluación y análisis de la información, que junto al conocimiento experiencial del profesional y las necesidades y características de la persona, busca encontrar soluciones atingentes a los problemas o situaciones que presenta el usuario (Tomlin \& Borgetto, 2011). Esta se conforma en su estructura más simple de cuatro componentes fundamentales: la evidencia científica disponible, la percepción y experiencia del profesional, la percepción y necesidades del usuario, y la situación contextual específica (Arbesman, Lieberman, \& Berlanstein, 2015). Este concepto puede parecer amedrentador a primera vista, puesto que la frase "basado en la evidencia" podría estar íntimamente relacionada a paradigmas positivistas de intervención en salud (paradigma que en ocasiones puede ser poco acogido por la disciplina), y que podría ser erróneamente interpretado como el uso exclusivo de la información científica para la toma de decisiones. A decir verdad, la palabra "evidencia" solo hace alusión a la literatura y experiencia científica disponible en relación a una determinada situación y que puede ser utilizada para la resolución de preguntas en un área o campo específico (Du Toit, Wilkinson, \& Adam, 2010)as in many other countries, the development of research capacity in students and their early professional career is regarded as of major importance. Within the context of clinical education for occupational therapy students at the University of the Free State, a lecturer and her students embarked on a collaborative journey while fulfilling the requirements of their undergraduate curriculum. The outcome is a model promoting evidence-based practice (EBP. Cuando incorporamos la palabra "evidencia" dentro del concepto de EBP, ésta pasa a ser solo un acápite de lo que significa este método (sumándose a los demás componentes que conforman la EBP: La percepción y experiencia del profesional, las necesidades del usuario y la situación contextual), y no se convierte bajo ninguna circunstancia en el único factor determinante para esclarecer una determina decisión en relación a un tratamiento o acción específica que se llevará a cabo (Gustafsson, Molineux, \& Bennett, 2014), sino que, se suma como un elemento que aporta a la visión y entendimiento que tenemos del problema. Por lo tanto no deja de ser fundamental que para comprender este método, debemos contemplar todos sus componentes de forma simétrica entendiendo que alguno de estos factores tomará mayor relevancia de acuerdo a la situación específica en la cual nos encontremos situados (Chabon, Morris, \& Lemoncello, 2011). Por otra parte, es determinante contemplar el carácter laxo que tiene la EBP como un proceso que está en un cambio continuo (comprendiendo que estos cuatro factores se encuentran en una constante renovación) y en una evaluación permanente (Thomas, Saroyan, \& Lajoie, 2012), que por ningún motivo debe ser entendida como una práctica lineal, debido a que su aplicación no garantiza resultados exitosos al primer intento, sino más bien busca generar un pensamiento y accionar crítico y reflexivo en relación a las propias praxis (Gustafsson et al., 2014).

En relación a la implementación de la TOBE, una situación que no deja de ser interesante, es el hecho de que la incorporación de la EBP en Terapia Ocupacional no solo es un desafío en lugares en donde el desarrollo de la disciplina es reciente, sino que también es un tópico de gran relevancia en contextos donde la práctica de la Terapia Ocupacional se encuentra más posicionada (Boruff \& Thomas, 2011), y en donde se han establecido esfuerzos para incrementar la incorporación de la EBP en el actuar cotidiano.

La especificidad en habilidades y conocimientos requeridos para la utilización de la EBP puede incidir en su baja utilización en Terapia Ocupacional, debido a que implica tener una base de conocimientos específicos mínimos, sobre todo en relación a la adecuada interpretación y búsqueda de la literatura científica (conocimientos que podrían estar escasamente incorporados en los Terapeutas Ocupacionales y en su formación profesional), así como un actuar y pensamiento multifactorial y moldeable, que implica reconocer que el propio conocimiento profesional corresponde solo a 
una parte de lo que es el razonamiento clínico y toma de decisiones en salud (Vogel, 2012).

Un factor que podría influir en la dificultad que presentan los Terapeutas Ocupacionales en la incorporación de la EBP a su práctica diaria es la información teórico-práctica incorporada durante su formación. Debido a que la praxis y entendimiento de la Terapia Ocupacional se fundamentan bajo el alero de la ciencia de la ocupación, área de la ciencia que es relativamente joven y que aún se encuentra en un proceso de solidificación de su identidad y dirección, muchas veces puede llevar a que la práctica busque respaldarse en todos sus ámbitos por teorías científicas correspondientes a otras ciencias, perdiéndose el foco central que tiene la ocupación, así como la definición propia de lo que es Terapia Ocupacional; o por el contrario, que la profesión tienda a convertirse en una disciplina más "técnica" que científica, donde se prioriza de sobremanera la acción basada en la experiencia práctica y los resultados subjetivos.

Aunque la investigación no es sinónimo de EBP, otro factor que determina la baja incorporación de la TOBE es la escases de investigaciones y literatura científica existente en relación a la práctica de la Terapia Ocupacional (Sudsawad, 2005). El hecho de que la praxis de la Terapia Ocupacional desde su esencia este empapada de una impronta que implica una modificación constante en relación a las necesidades que cada sujeto va presentando en su contexto socio-histórico determinado, y que no tiene un carácter rígido vinculado a una manifestación clínica determinada, puede tomarse como una de las mayores limitantes para la generación de evidencia y el uso de la EBP en el ejercicio diario. Esto, eventualmente dificultaría en gran medida la replicabilidad y estandarización de los procesos, haciéndolos poco homologables a experiencias probadas en contextos diferentes. No obstante, en los últimos años existe investigación creciente en relación a modelos de atención centrados en la persona o en las necesidades de los usuarios, y cuyo distintivo es el ajuste constante del tratamiento a las necesidades que el propio sujeto va manifestando, y su carácter psicosocial, incluso incorporando al ambiente como parte del tratamiento (Dwamena et al, 2012). Si bien, la gran debilidad de estos modelos son las dificultades metodológicas que eventualmente pueden presentar para su estudio, la evidencia existente ha mostrado resultados alentadores en términos de la mejoría de la calidad de vida y bienestar del usuario, así como otros parámetros de importancia clínica (Olsson, Jakobsson Ung, Swedberg, \& Ekman,
2013). Entonces, si modelos que pueden parecer complejos de investigar como la atención centrada en la persona han presentado un número creciente de publicaciones y replicaciones en diversos contextos, ¿Por qué aun cuesta tanto incrementar la investigación en Terapia Ocupacional?, ¿Por qué es todo un desafío incorporar los nuevos hallazgos científicos inherentes a la ocupación y la Terapia Ocupacional en la praxis diaria de la profesión?

La evidencia no solo se construye de la práctica científica sino que de la experiencia del propio profesional, unificándose constantemente ambos criterios, Esto puesto que la práctica le da un sentido a la ciencia y la ciencia le da sentido a la práctica, ambas adaptándose constantemente a las características y necesidades de la persona, quien debe ser el motor fundamental de toda práctica en salud.

La incorporación de la EBP en la Terapia Ocupacional no solo es importante para mejorar la práctica e identidad de la disciplina, sino que aun más importante es la responsabilidad que se tiene con los usuarios de ofrecer y entregar la mejor atención, herramientas y guía ante su situación o dificultad específica (Clair \& Newcombe, 2014). Continuar perpetuando prácticas y estrategias que fueron desarrolladas hace 30 o 40 años en un contexto socio-histórico diferente al actual sin ponerlas a prueba de forma crítica y reflexiva puede ser una acción negligente hacia el usuario y su salud, entendiendo que los conocimientos y avances en salud se encuentran en constante renovación (por lo menos, cada 5 años), y que como profesionales que trabajan con los individuos y sus situaciones de vida se tiene una obligación de mantenerse en una constante actualización y reflexión en relación a los nuevos hallazgos y avances en la intervención en salud, con el fin de entregar y ofrecer la mejor y más atingente atención en pro de mantener o mejorar la calidad de vida de las personas, quienes finalmente son los actores principales de toda práctica. 


\section{RefERENCIAS}

Arbesman, M., Lieberman, D., \& Berlanstein, D.R. (2015). Method for the evidence-based reviews on occupational therapy and stroke. The American Journal of Occupational Therapy, 69(1), 6901180020p15. doi: 10.5014/ajot.2015.013524.

Boruff, J. T., \& Thomas, A. (2011). Integrating evidence-based practice and information literacy skills in teaching physical and occupational therapy students. Health Information and Libraries Journal, 28(4), 264-272. doi:10.1111/j.1471-1842.2011.00953.x

Chabon, S., Morris, J., \& Lemoncello, R. (2011). Ethical deliberation: a foundation for evidence-based practice. Seminars in Speech Language, 32(4), 298-308. doi: 10.1055/s-0031-1292755.

Clair, V. a. W.-S., \& Newcombe, D. B. (2014). Values and ethics in practice-based decision making: Les valeurs et l'ethique lors des prises de decision dans la pratique. Canadian Journal of Occupational Therapy, 81(3), 154-162. doi:10.1177/0008417414535083

Cohn, E.S., Coster, W.J., \& Kramer, J.M. (2014). Facilitated learning model to teach habits of evidence-based reasoning across an integrated master of science in occupational therapy curriculum. The American Journal of Occupational Therapy, 68(2), S73-82. doi: 10.5014/ ajot.2014.685S05.

Dwamena, F., Holmes-Rovner, M., Gaulden, C.M., Jorgenson, S., Sadigh, G., Sikorskii, A., Lewin, S., Smith, R.C., Coffey, J., \& Olomu, A. (2012). Interventions for providers to promote a patient-centred approach in clinical consultations. The Cochrane Database of Systematic Reviews, 12(12), CD003267. doi: 10.1002/14651858. CD003267.

Du Toit, S. H. J., Wilkinson, A. C., \& Adam, K. (2010). Role of research in occupational therapy clinical practice: Applying action learning and action research in pursuit of evidence-based practice. Australian Occupational Therapy Journal, 57(5), 318-330. doi:10.1111/ j.1440-1630.2010.00851.x

Evidence-Based Medicine Working Group. (1992). Evidence-based medicine. A new approach to teaching the practice of medicine. JAMA, 268(17), 2420-5.

Gustafsson, L., Molineux, M., \& Bennett, S. (2014). Contemporary occupational therapy practice: The challenges of being evidence based and philosophically congruent. Australian Occupational Therapy Journal, 121-123. doi:10.1111/1440-1630.12110

Olsson, L.-E., Jakobsson Ung, E., Swedberg, K., \& Ekman, I. (2013). Efficacy of person-centred care as an intervention in controlled trials - a systematic review. Journal of Clinical Nursing, 22(3-4), 456-65. doi:10.1111/jocn.12039

Sudsawad, P. (2005). A Conceptual Framework To Increase Usability of Outcome Research for Evidence-Based Practice. The American Journal of Occupational Therapy : Official Publication of the American Occupational Therapy Association, 59(1998), 351-355. Retrieved from http://ajot.aota.org/article.aspx?articleid=1871712
Thomas, A., Saroyan, A., \& Lajoie, S.P. (Creation of an evidence-based practice reference model in falls prevention: findings from occupational therapy. Disability and Rehabilitation, 34(4), 311-28. doi: 10.3109/09638288.2011.607210.

Tomlin, G., \& Borgetto, B. (2011). Research Pyramid: a new evidencebased practice model for occupational therapy. The American Journal of Occupational Therapy 65(2):189-96. Retrived from http://ajot.aota.org/article.aspx? volume=65\&page $=189$

Upton, D., Stephens, D., Williams, B., \& Scurlock-Evans, L. (2014). Occupational therapists' attitudes, knowledge, and implementation of evidence-based practice: a systematic review of published research. The British Journal of Occupational Therapy, 77(1), 24-38. doi:10.4276/030802214X13887685335544

Vogel, K. a. (2012). Librarians and occupational therapy faculty: A Collaboration for teaching evidence-based practice. Journal of Allied Health, 41(1). 